Constraints in the vari at i onal princi ple for st at i onary st at es

\begin{tabular}{|l|l|}
\hline 著者 & OHTA Kat suhi sa \\
\hline $\begin{array}{l}\text { j our nal or } \\
\text { publ i cat i on } \mathrm{titl} \text { e }\end{array}$ & Jour nal of Nat hemat i cal Chemi st ry \\
\hline vol une & 56 \\
\hline number & 3 \\
\hline page range & $813-824$ \\
\hline year & $2018-03$ \\
\hline URL & ht t p: //hdl . handl e. net /10258/00009916 \\
\hline
\end{tabular}




\title{
Constraints in the variational principle for stationary states
}

\author{
Katsuhisa Ohta $^{1, *}$ \\ 1 Applied Chemistry Research Unit, Graduate School of Engineering, \\ Muroran Institute of Technology, Hokkaido, 050-8585, Japan
}

\begin{abstract}
Constraints in the variational principle for stationary states (VPSS) are classified in accordance with Dirac's constrained classical mechanics and the time-dependent variational principle (TDVP). All of the VPSS constraints are required to belong to the first-class TDVP as constants of motion to ensure the real-valuedness of the Lagrange multipliers. The VPSS constraints are further classified as either first-class or second-class. The first-class VPSS constraints are constants of variation with symmetry-adapted wave functions. If the representation basis for the constraint operators is incomplete, however, the first-class VPSS constraints lead to broken-symmetry solutions. The nondegenerate energies of ${ }^{2} E^{\prime}$ at the $D_{3 h}$ geometry in the Jahn-Teller distortion of $\mathrm{H}_{3}$ are presented as an example of a problem with broken-symmetry. An ad hoc adjustment is suggested by considering the second-class pseudo-VPSS constraints with new adiabatic parameters.
\end{abstract}

PACS numbers:

*Electronic address: ohta@mmm.muroran-it.ac.jp 


\section{INTRODUCTION}

Constraints in the variational principle for stationary states (VPSS)[1] can be used for various purposes, such as to keep some formal symmetries of the system, to construct models of some physical situations, and to analyze physical or chemical consequences of some freedoms [2-5]. In previous works [6-9], by using the pseudo-classical structure of the timedependent variational principle (TDVP)[10-17], we have systematically analyzed nonsingular constraints in the TDVP in accordance with Dirac's constrained classical mechanics $[18-21]$.

In this investigation, the VPSS constraints are also classified in a similar manner to these nonsingular constraints. In $\S I I$, the VPSS with nonsingular constraints is formulated by the Lagrange multiplier method. In $§ I I I$, the VPSS constraints, including singular constraints, are further classified as either first-class or second-class. If the representation basis for the constraint operators is incomplete, the first-class VPSS constraints lead to solutions related to broken-symmetry. In $\S \mathrm{IV}$, as an example of a Hamiltonian problem corresponding to broken-symmetry, nondegenerate energies associated with ${ }^{2} E^{\prime}$ of the $D_{3 h}$ geometry in the Jahn-Teller distortion of $\mathrm{H}_{3}$ were studied. $\S \mathrm{V}$ gives a summary of the present work.

\section{VPSS WITH CONSTRAINTS BY THE LAGRANGE MULTIPLIER METHOD}

First we assume a normalization condition for the wave function of the VPSS as

$$
\langle\Psi \mid \Psi\rangle-1=0
$$

Other constraints are also introduced through the expectation values of Hermitian operators $\left\{\hat{g}_{i}\right\}_{i=1, M}$ with corresponding real values $\left\{g_{i}^{0}\right\}_{i=1, M}$ as

$$
\left\langle\Psi\left|\hat{G}_{i}\right| \Psi\right\rangle=\left\langle\Psi\left|\left(\hat{g}_{i}-g_{i}^{0}\right)\right| \Psi\right\rangle=0, \quad(i=1, \cdots, M)
$$

where the value $g_{i}^{0}$ is an internal dividing point of the eigenvalues of $\hat{g}_{i}$, as shown in Appendix A. The functional $I$ of the VPSS with real Lagrange multipliers is written

$$
I\left[\Psi^{*}, \Psi\right]=\langle\Psi|\hat{H}| \Psi\rangle-\lambda_{0}(\langle\Psi \mid \Psi\rangle-1)-\sum_{i=1}^{M} \lambda_{i}\left\langle\Psi\left|\hat{G}_{i}\right| \Psi\right\rangle .
$$


Euler's equation as the necessary condition for the extremal of the functional $I$ is written

$$
\hat{H}|\Psi\rangle-\lambda_{0}|\Psi\rangle-\sum_{i=1}^{M} \lambda_{i} \hat{G}_{i}|\Psi\rangle=0 .
$$

By taking inner products of Eq. (4) first with $\langle\Psi|$, we have

$$
\lambda_{0}=\langle\Psi|\hat{H}| \Psi\rangle \text {, }
$$

and second with $\left\langle\hat{G}_{j} \Psi\right|$, we obtain the inhomogeneous linear equations for the Lagrange multipliers,

$$
\left\langle\Psi\left|\hat{G}_{j} \hat{H}\right| \Psi\right\rangle-\sum_{i=1}^{M}\left\langle\Psi\left|\hat{G}_{j} \hat{G}_{i}\right| \Psi\right\rangle \lambda_{i}=0 .
$$

In matrix form $(\boldsymbol{h})_{j}=\left\langle\Psi\left|\hat{G}_{j} \hat{H}\right| \Psi\right\rangle,(\boldsymbol{S})_{j i}=\left\langle\Psi\left|\hat{G}_{j} \hat{G}_{i}\right| \Psi\right\rangle$, and $(\boldsymbol{\lambda})_{i}=\lambda_{i}$, Eq. (6) is rewritten simply as

$$
h-S \lambda=0 .
$$

If the Hermitian matrix $\boldsymbol{S}$ is nonsingular, we have directly

$$
\lambda_{i}=\sum_{j=1}^{M}\left(\boldsymbol{S}^{-1}\right)_{i j}\left\langle\Psi\left|\hat{G}_{j} \hat{H}\right| \Psi\right\rangle .
$$

If we substitute the obtained multipliers (8) into Eq. (4), we have

$$
\hat{H}_{D}|\Psi\rangle=\lambda_{0}|\Psi\rangle
$$

thereby recovering the usual Schrödinger equation. The Hamiltonian $\hat{H}_{D}$ is defined as

$$
\hat{H}_{D}=\hat{H}-\sum_{i=1}^{M} \sum_{j=1}^{M} \hat{G}_{i}\left(\boldsymbol{S}^{-1}\right)_{i j}\left\langle\Psi\left|\hat{G}_{j} \hat{H}\right| \Psi\right\rangle,
$$

which is similar to Dirac's constrained Hamiltonian in classical mechanics[18, 19]. We can also rewrite Eq. (4) with projection operators as

$$
\left[\hat{1}-|\Psi\rangle\langle\Psi|-\hat{P}^{G \Psi}\right] \hat{H}|\Psi\rangle=0
$$

where the projection operator $\hat{P}^{G \Psi}$ is defined as

$$
\hat{P}^{G \Psi}=\sum_{i=1}^{M} \sum_{j=1}^{M}\left|\hat{G}_{i} \Psi\right\rangle\left(\boldsymbol{S}^{-1}\right)_{i j}\left\langle\hat{G}_{j} \Psi\right| .
$$

The proof of the equivalence of Eq. (11) and the direct VPSS within the projected space is given in Appendix B. 


\section{CLASSIFICATION OF VPSS CONSTRAINTS}

In this section, we analyze in complete generality the nonsingularity of the coefficient matrix $\boldsymbol{S}$ in Eq. (7) and further classify the VPSS constraints as first-class and second-class.

A. All of the VPSS constraints belong to the first-class TDVP constraints as constants of motion

To maintain the real-valuedness of the Lagrange multipliers in the functional $I$ of Eq. (3), we restrict the vector $\boldsymbol{h}$ and the matrix $\boldsymbol{S}$ in Eq. (7) to be real, i.e.,

$$
\left\langle\Psi\left|\hat{G}_{i} \hat{H}\right| \Psi\right\rangle=\left\langle\Psi\left|\hat{G}_{i} \hat{H}\right| \Psi\right\rangle^{*}, \quad\left\langle\Psi\left|\hat{G}_{i} \hat{G}_{j}\right| \Psi\right\rangle=\left\langle\Psi\left|\hat{G}_{i} \hat{G}_{j}\right| \Psi\right\rangle^{*}
$$

are sufficient conditions. Because the equation $\langle\Psi|\hat{A} \hat{B}| \Psi\rangle^{*}=\langle\Psi|\hat{B} \hat{A}| \Psi\rangle$ holds for Hermitian operators $\hat{A}$ and $\hat{B}$ generally, Eq. (13) leads directly to

$$
\left\langle\Psi\left|\left[\hat{H}, \hat{G}_{i}\right]\right| \Psi\right\rangle=0, \quad\left\langle\Psi\left|\left[\hat{G}_{i}, \hat{G}_{j}\right]\right| \Psi\right\rangle=0
$$

By Eq. (14), the VPSS constraints operators $\left\{\hat{G}_{i}\right\}_{i=1, M}$ are classified as first-class TDVP operators[6-9] in the form of the Hypervirial Theorem[22, 23]. The VPSS has already been derived so that trajectories do not evolve with time within the framework of the TDVP $[7,8]$. Thus, as statics is a special case of dynamics in classical mechanics, it may be reasonable that all of the VPSS constraints belong to the first-class TDVP as constants of motion.

\section{B. Classification of VPSS constraints into first-class and second-class}

We now classify the VPSS constraints, including those that are singular in Eq. (7). The inhomogeneous linear equation (7) is rewritten

$$
\left(\begin{array}{c}
\left\langle\hat{G}_{1} \Psi \mid \hat{H} \Psi\right\rangle \\
\vdots \\
\left\langle\hat{G}_{M} \Psi \mid \hat{H} \Psi\right\rangle
\end{array}\right)-\left(\begin{array}{ccc}
\left\langle\hat{G}_{1} \Psi \mid \hat{G}_{1} \Psi\right\rangle & \cdots & \left\langle\hat{G}_{1} \Psi \mid \hat{G}_{M} \Psi\right\rangle \\
\vdots & \ddots & \vdots \\
\left\langle\hat{G}_{M} \Psi \mid \hat{G}_{1} \Psi\right\rangle & \cdots & \left\langle\hat{G}_{M} \Psi \mid \hat{G}_{M} \Psi\right\rangle
\end{array}\right)\left(\begin{array}{c}
\lambda_{1} \\
\vdots \\
\lambda_{M}
\end{array}\right)=\left(\begin{array}{c}
0 \\
\vdots \\
0
\end{array}\right) .
$$


We first diagonalize the coefficient matrix $\boldsymbol{S}$ using an orthogonal matrix $\boldsymbol{T}$ as

$$
\begin{aligned}
& \boldsymbol{D}=\boldsymbol{T}^{t} \boldsymbol{S} \boldsymbol{T} \\
& =\left(\begin{array}{ccc|ccc}
\left\langle\hat{G}_{1}^{(2)} \Psi \mid \hat{G}_{1}^{(2)} \Psi\right\rangle & \cdots & \left\langle\hat{G}_{1}^{(2)} \Psi \mid \hat{G}_{M^{(2)}}^{(2)} \Psi\right\rangle & \left\langle\hat{G}_{1}^{(2)} \Psi \mid \hat{G}_{1}^{(1)} \Psi\right\rangle & \cdots & \left\langle\hat{G}_{1}^{(2)} \Psi \mid \hat{G}_{M-M^{(2)}}^{(1)} \Psi\right\rangle \\
\vdots & \ddots & \vdots & \vdots & \ddots & \vdots \\
\left\langle\hat{G}_{M^{(2)}}^{(2)} \Psi \mid \hat{G}_{1}^{(2)} \Psi\right\rangle & \cdots & \left\langle\hat{G}_{M^{(2)}}^{(2)} \Psi \mid \hat{G}_{M^{(2)}}^{(2)} \Psi\right\rangle & \left\langle\hat{G}_{M^{(2)}}^{(2)} \Psi \mid \hat{G}_{1}^{(1)} \Psi\right\rangle & \cdots & \left\langle\hat{G}_{M^{(2)}}^{(2)} \Psi \mid \hat{G}_{M-M^{(2)}}^{(1)} \Psi\right\rangle \\
\hline\left\langle\hat{G}_{1}^{(1)} \Psi \mid \hat{G}_{1}^{(2)} \Psi\right\rangle & \cdots & \left\langle\hat{G}_{1}^{(1)} \Psi \mid \hat{G}_{M^{(2)}}^{(2)} \Psi\right\rangle & \left\langle\hat{G}_{1}^{(1)} \Psi \mid \hat{G}_{1}^{(1)} \Psi\right\rangle & \cdots & \left\langle\hat{G}_{1}^{(1)} \Psi \mid \hat{G}_{M-M^{(2)}}^{(1)} \Psi\right\rangle \\
\vdots & \ddots & \vdots & \vdots & \ddots & \vdots \\
\left\langle\hat{G}_{M-M^{(2)}}^{(1)} \Psi \mid \hat{G}_{1}^{(2)} \Psi\right\rangle & \cdots & \left\langle\hat{G}_{M-M^{(2)}}^{(1)} \Psi \mid \hat{G}_{M^{(2)}}^{(2)} \Psi\right\rangle & \left\langle\hat{G}_{M-M^{(2)}}^{(1)} \Psi \mid \hat{G}_{1}^{(1)} \Psi\right\rangle & \cdots & \left\langle\hat{G}_{M-M^{(2)}}^{(1)} \Psi \mid \hat{G}_{M-M^{(2)}}^{(1)} \Psi\right\rangle
\end{array}\right) \\
& =\left(\begin{array}{ccc|ccc}
d_{1} & 0 & 0 & 0 & \cdots & 0 \\
0 & \ddots & \vdots & \vdots & \cdots & \vdots \\
0 & \cdots & d_{M^{(2)}} & 0 & \cdots & 0 \\
\hline 0 & \cdots & 0 & 0 & \cdots & 0 \\
0 & \cdots & 0 & 0 & \cdots & 0 \\
0 & \cdots & 0 & 0 & \cdots & 0
\end{array}\right)
\end{aligned}
$$

The rank of $\boldsymbol{S}$ is found to be $M^{(2)}$ with all eigenvalues positive,

$$
d_{a}>0, \quad\left(a=1, \ldots, M^{(2)}\right) .
$$

The transformed vectors under $\boldsymbol{T}^{t}$ are written

$$
\left(\begin{array}{c}
\left\langle\hat{G}_{1}^{(2)} \Psi\right| \\
\vdots \\
\frac{\left\langle\hat{G}_{M^{(2)}}^{(2)} \Psi\right|}{\left\langle\hat{G}_{1}^{(1)} \Psi\right|} \\
\vdots \\
\left\langle\hat{G}_{M-M^{(2)}}^{(1)} \Psi\right|
\end{array}\right)=\boldsymbol{T}^{t}\left(\begin{array}{c}
\left\langle\hat{G}_{1} \Psi\right| \\
\vdots \\
\left\langle\hat{G}_{M} \Psi\right|
\end{array}\right), \quad\left(\begin{array}{c}
\lambda_{1}^{(2)} \\
\vdots \\
\frac{\lambda_{M^{(2)}}^{(2)}}{\lambda_{1}^{(1)}} \\
\vdots \\
\lambda_{M-M^{(2)}}^{(1)}
\end{array}\right)=\boldsymbol{T}^{t}\left(\begin{array}{c}
\lambda_{1} \\
\vdots \\
\lambda_{M}
\end{array}\right)
$$

Because the Hermitian norm is non-negative, the diagonal elements $\left\{\left\langle\hat{G}_{b}^{(1)} \Psi \mid \hat{G}_{b}^{(1)} \Psi\right\rangle=0\right\}_{b=1, M-M^{(2)}}$ in Eq. (16) lead to zero vectors

$$
\left|\hat{G}_{b}^{(1)} \Psi\right\rangle=0, \quad\left(b=1, \cdots, M-M^{(2)}\right) .
$$

We classify these components as first-class VPSS constraints. The transformed equation of 
Eq. (15) is written simply as

$$
\left(\begin{array}{c}
\left\langle\hat{G}_{1}^{(2)} \Psi \mid \hat{H} \Psi\right\rangle \\
\vdots \\
\left\langle\hat{G}_{M^{(2)}}^{(2)} \Psi \mid \hat{H} \Psi\right\rangle \\
0 \\
\vdots \\
0
\end{array}\right)-\left(\begin{array}{ccc|ccc}
d_{1} & 0 & 0 & 0 & \cdots & 0 \\
0 & \ddots & \vdots & \vdots & \cdots & \vdots \\
0 & \cdots & d_{M^{(2)}} & 0 & \cdots & 0 \\
\hline 0 & \cdots & 0 & 0 & \cdots & 0 \\
0 & \cdots & 0 & 0 & \cdots & 0 \\
0 & \cdots & 0 & 0 & \cdots & 0
\end{array}\right)\left(\begin{array}{c}
\lambda_{1}^{(2)} \\
\vdots \\
\lambda_{M^{(2)}}^{(2)} \\
\lambda_{1}^{(1)} \\
\vdots \\
\lambda_{M-M^{(2)}}^{(1)}
\end{array}\right)=\left(\begin{array}{c}
0 \\
\vdots \\
0
\end{array}\right)
$$

where the inhomogeneous terms $\left\{\left\langle\hat{G}_{b}^{(1)} \Psi \mid \hat{H} \Psi\right\rangle\right\}_{b=1, M-M^{(2)}}$ disappear with Eq. (19). The Lagrange multipliers $\left\{\lambda_{b}^{(1)}\right\}_{b=1, M-M^{(2)}}$ for the first-class VPSS constraints become arbitrary as for the first-class TDVP constraints[6, 7]. In contrast, the multipliers $\left\{\lambda_{a}^{(2)}\right\}_{a=1, M^{(2)}}$ for $\left\{\hat{G}_{a}^{(2)}\right\}_{a=1, M^{(2)}}$ components are uniquely determined,

$$
\lambda_{a}^{(2)}=\frac{\left\langle\hat{G}_{a}^{(2)} \Psi \mid \hat{H} \Psi\right\rangle}{d_{a}}, \quad\left(a=1, \ldots, M^{(2)}\right) .
$$

We call these $\left\{\hat{G}_{a}^{(2)}\right\}_{a=1, M^{(2)}}$ components second-class VPSS constraints. The Lagrange multipliers in Eq. (8) can be obtained from these $\left\{\lambda_{a}^{(2)}\right\}_{a=1, M^{(2)}}$ by the inverse transformation of Eq. (18).

\section{The first-class VPSS constraints as constants of variation}

If we have a linear equation of the constraint operators $\left\{\hat{G}_{i}\right\}_{i=1, M}$

$$
\hat{F}\left(\hat{G}_{1}, \ldots \hat{G}_{M}\right)=\sum_{i=1}^{M} \alpha_{i} \hat{G}_{i}=0
$$

the operator $\hat{G}_{b}^{(1)}=\hat{F}$ induces a singularity in Eq. (20) with a trivial zero vector

$$
\left|\hat{G}_{b}^{(1)} \Psi\right\rangle=\sum_{i=1}^{M} \alpha_{i}\left|\hat{G}_{i} \Psi\right\rangle=0 \text {. }
$$

Apart from such trivial zero vectors, the genuine first-class constraint operator $\hat{G}_{b}^{(1)}=\hat{g}_{b}^{(1)}-$ $g_{b}^{0}$ in Eq. (19) leads to the eigenvalue equation

$$
\hat{g}_{b}^{(1)}|\Psi\rangle=g_{b}^{0}|\Psi\rangle
$$


Hence the wave function $|\Psi\rangle$ is a symmetry-adapted solution if the operator $\hat{g}_{b}^{(1)}$ commutes with $\hat{H}$. The expectation value of $\hat{g}_{b}^{(1)}$, of course, does not change as it is a so-called constant of variation.

The classification of the VPSS constraints is summarized in Table I with that of Dirac's constrained classical mechanics and the TDVP.

\section{SECOND-CLASS PSEUDO-VPSS CONSTRAINTS FOR BROKEN- SYMMETRY PROBLEMS}

The genuine first-class constraint operators $\left\{\hat{g}_{b}^{(1)}\right\}_{b=1, d}$, which commute with $\hat{H}$, satisfy the eigenvalue equations given in Eq. (24). These first-class constraints, however, sometimes lead to solutions related to broken-symmetry because the representation basis for the constraint operators is incomplete. In such cases, we can consider the second-class pseudo-VPSS constraints

$$
\left\langle\Psi\left|\left(\hat{g}_{b}^{(1)}-g_{b}^{0}\right)\right| \Psi\right\rangle=0, \quad(b=1, \ldots, d),
$$

although these constraints are just necessary conditions of the eigenvalue equations. The value $g_{b}^{0}$ should be an internal dividing point of the eigenvalues of $\hat{g}_{b}^{(1)}$ as shown in Appendix A. In this section, we give an example of a Hamiltonian problem corresponding to brokensymmetry.

\section{A. Nondegenerate energies of ${ }^{2} E^{\prime}$ at the $D_{3 h}$ geometry in the Jahn-Teller distortion} of $\mathbf{H}_{3}$

Here we consider the Jahn-Teller distortion of $\mathrm{H}_{3}$ which is illustrated in Fig. 1. The height of the triangle is distorted from $0.730538 \AA\left(=\frac{\sqrt{3}}{2} R_{H H}\right)$ at $D_{3 h}$, where $R_{H H}$ is $0.843553 \AA$ obtained by the geometry optimization for $\mathrm{H}_{3}^{+}$at $D_{3 h}$ with RHF/STO-6G $[24,25]$. The point group symmetry of $\hat{H}$ changes in the Jahn-Teller distortion from $D_{3 h}$ to $C_{2 v}$. All the irreducible representation spaces at the $C_{2 v}$ geometries are one-dimensional. For the $D_{3 h}$ geometry, however, the two-dimensional irreducible representation space ${ }^{2} E^{\prime}$ should be constructed with degenerate energy levels. Open-shell Hartree-Fock wave functions UHF/DZV and ROHF/DZV[25], however, fail to obtain degenerate energy levels for ${ }^{2} E^{\prime}$ of the $D_{3 h}$ 
geometry (Fig. 2). The variational freedom within the Brillouin Theorem[24] of those wave functions is incomplete in the variational space constructed with the basis set, DZV. In contrast, the full-CI wave function, which is complete in the given variational space, can obtain the degenerate energies of ${ }^{2} E^{\prime}$ (Fig. 3).

\section{B. Ad hoc adjustment for the energy splitting of $\mathbf{H}_{3}$ at the $D_{3 h}$ geometry}

Here we suggest an ad hoc adjustment of the second-class pseudo-VPSS constraints (25) and a new adiabatic parameter to reconstruct the degenerate energy levels for the $D_{3 h}$ geometry. We use an even-averaged density matrix

$$
\rho=\frac{1}{2}\left|\Psi_{x}\right\rangle\left\langle\Psi_{x}\left|+\frac{1}{2}\right| \Psi_{y}\right\rangle\left\langle\Psi_{y}\right|
$$

where the two-dimensional $(x, y)$ coordinates are defined in Fig. 1. We can assign the two irreducible representation bases $\left\{\Psi_{x}, \Psi_{y}\right\}$ for the ${ }^{2} E^{\prime}$ of $D_{3 h}$ with the subgroup $C_{2 v}$ as $\Psi_{x}\left(b_{2}\right)$ and $\Psi_{y}\left(a_{1}\right)$. The expectation values of the electric moment operator $\hat{\boldsymbol{r}}=(\hat{x}, \hat{y})$ are

$$
\left\langle\Psi_{x}\left(b_{2}\right)\left|\hat{x}\left(b_{2}\right)\right| \Psi_{x}\left(b_{2}\right)\right\rangle=0, \quad\left\langle\Psi_{y}\left(a_{1}\right)\left|\hat{x}\left(b_{2}\right)\right| \Psi_{y}\left(a_{1}\right)\right\rangle=0,
$$

and

$$
\left\langle\Psi_{x}\left(b_{2}\right)\left|\hat{y}\left(a_{1}\right)\right| \Psi_{x}\left(b_{2}\right)\right\rangle=\alpha \neq 0, \quad\left\langle\Psi_{y}\left(a_{1}\right)\left|\hat{y}\left(a_{1}\right)\right| \Psi_{y}\left(a_{1}\right)\right\rangle=\beta \neq 0,
$$

with $C_{2 v}$ point symmetry. Next we assume that the averaged moment in the two-dimensional degenerate space ${ }^{2} E^{\prime}$ disappears. Hence we have

$$
\begin{aligned}
& \operatorname{Tr}[\rho \hat{x}]=\frac{1}{2}\left\langle\Psi_{x}\left(b_{2}\right)\left|\hat{x}\left(b_{2}\right)\right| \Psi_{x}\left(b_{2}\right)\right\rangle+\frac{1}{2}\left\langle\Psi_{y}\left(a_{1}\right)\left|\hat{x}\left(b_{2}\right)\right| \Psi_{y}\left(a_{1}\right)\right\rangle=0, \\
& \operatorname{Tr}[\rho \hat{y}]=\frac{1}{2}\left\langle\Psi_{x}\left(b_{2}\right)\left|\hat{y}\left(a_{1}\right)\right| \Psi_{x}\left(b_{2}\right)\right\rangle+\frac{1}{2}\left\langle\Psi_{y}\left(a_{1}\right)\left|\hat{y}\left(a_{1}\right)\right| \Psi_{y}\left(a_{1}\right)\right\rangle=\frac{1}{2}(\alpha+\beta)=0,
\end{aligned}
$$

and

$$
\beta=-\alpha
$$

Now we can set the second-class pseudo-VPSS constraints (25) for $\left\{\Psi_{x}, \Psi_{y}\right\}$ as

$$
\begin{array}{ll}
\left\langle\Psi_{x}|\hat{x}| \Psi_{x}\right\rangle=0, \quad\left\langle\Psi_{x}|\hat{y}| \Psi_{x}\right\rangle=\alpha, \\
\left\langle\Psi_{y}|\hat{x}| \Psi_{y}\right\rangle=0, \quad\left\langle\Psi_{y}|\hat{y}| \Psi_{y}\right\rangle=-\alpha,
\end{array}
$$


where we consider $\alpha$ as one of the adiabatic parameters in the VPSS calculations. The VPSS functionals are defined as

$$
\begin{aligned}
& I_{x}=\left\langle\Psi_{x}|\hat{H}| \Psi_{x}\right\rangle-\lambda_{0}\left(\left\langle\Psi_{x} \mid \Psi_{x}\right\rangle-1\right)-\lambda_{1}\left\langle\Psi_{x}|(\hat{x}-0)| \Psi_{x}\right\rangle-\lambda_{2}\left\langle\Psi_{x}|(\hat{y}-\alpha)| \Psi_{x}\right\rangle, \\
& I_{y}=\left\langle\Psi_{y}|\hat{H}| \Psi_{y}\right\rangle-\lambda_{0}^{\prime}\left(\left\langle\Psi_{y} \mid \Psi_{y}\right\rangle-1\right)-\lambda_{1}^{\prime}\left\langle\Psi_{y}|(\hat{x}-0)| \Psi_{y}\right\rangle-\lambda_{2}^{\prime}\left\langle\Psi_{y}|(\hat{y}+\alpha)| \Psi_{y}\right\rangle .
\end{aligned}
$$

By performing the VPSS calculations with $I_{x}$ and $I_{y}$ independently, we obtain two extremal energies

$$
E_{x}(\alpha)=\left\langle\Psi_{x}(\alpha)|\hat{H}| \Psi_{x}(\alpha)\right\rangle, \quad E_{y}(\alpha)=\left\langle\Psi_{y}(\alpha)|\hat{H}| \Psi_{y}(\alpha)\right\rangle
$$

as functions of adiabatic parameter $\alpha$. Finally, we search for a critical point $\alpha^{\sharp}$ for parameter $\alpha$, where the adiabatic energies are degenerate,

$$
\left.E_{x}(\alpha)\right|_{\alpha=\alpha^{\sharp}}=\left.E_{y}(\alpha)\right|_{\alpha=\alpha^{\sharp}} .
$$

Unfortunately, however, the consistency of $\alpha$ with other adiabatic parameters cannot be guaranteed in general. For example, the degenerate energy of Eq. (37) is not necessarily continuous along the nuclear coordinate in the Jahn-Teller distortion from a $D_{3 h}$ geometry to that of $C_{2 v}$. The ad hoc adjustment is similar to Roothaan's method for the spin-restricted open-shell Hartree-Fock theory(ROHF)[26]. Roothaan's method introduces a fractional occupation number which is averaged over the degenerate orbitals instead of the state average with the total wave functions as given in Eq. (26).

\section{SUMMARY}

All of the VPSS constraints are required to belong to the first-class TDVP constraints as constants of motion to ensure the real-valuedness of the Lagrange multipliers. According to the singularity analysis for the determining equation of the Lagrange multipliers, the constraints in the VPSS are classified as first-class or second-class. The singular constraints are identified as first-class in which the wave function is an eigenfunction of the constraint operators. If the first-class VPSS constraint operators commute with $\hat{H}$, they are related to the constants of variation with symmetry-adapted wave functions. However, if the representation basis for the constraint operators is incomplete, the first-class VPSS constraints lead to solutions corresponding to broken-symmetry. An ad hoc adjustment for such problems 
with broken-symmetry is suggested by considering the second-class pseudo-VPSS constraints with new adiabatic parameters. 


\section{Appendices}

\section{Appendix A: Constraints to internally dividing points of eigenvalues}

In Eq. (2), we set the VPSS constraint operator $\hat{g}$ with an appropriate real value $g^{0}$ as

$$
\hat{G}=\hat{g}-g^{0}, \quad\langle\Psi|\hat{G}| \Psi\rangle=0 .
$$

If we expand the wave function $|\Psi\rangle$ with the complete and orthonormal eigenfunctions of $\hat{g}$ as $\left\{\hat{g}\left|g_{i}\right\rangle=g_{i}\left|g_{i}\right\rangle\right\}_{i=1, K}$, we have

$$
|\Psi\rangle=\left(\sum_{i=1}^{K}\left|g_{i}\right\rangle\left\langle g_{i}\right|\right)|\Psi\rangle=\sum_{i=1}^{K} c_{i}\left|g_{i}\right\rangle .
$$

The expectation value with respect to $\Psi$ is written

$$
\langle\Psi|\hat{G}| \Psi\rangle=\sum_{i=1}^{K}\left|c_{i}\right|^{2} g_{i}-g^{0} \sum_{i=1}^{K}\left|c_{i}\right|^{2}=0
$$

The constraint value $g^{0}$ is expressed as

$$
g^{0}=\frac{\sum_{i=1}^{K}\left|c_{i}\right|^{2} g_{i}}{\sum_{i=1}^{K}\left|c_{i}\right|^{2}}
$$

The value $g^{0}$ for the constraint (A1) must be an internal dividing point of the eigenvalues.

\section{Appendix B: VPSS in the constrained space via projection operators}

We here consider the VPSS calculation in the constrained space directly using projection operators. By the normalization condition (1) and the constraints (2), we have

$$
\begin{aligned}
\delta\langle\Psi \mid \Psi\rangle & =\langle\delta \Psi \mid \Psi\rangle+\langle\Psi \mid \delta \Psi\rangle=0, \\
\delta\left\langle\Psi\left|\hat{G}_{i}\right| \Psi\right\rangle & =\left\langle\delta \Psi \mid \hat{G}_{i} \Psi\right\rangle+\left\langle\hat{G}_{i} \Psi \mid \delta \Psi\right\rangle=0, \quad(i=1, \ldots, M) .
\end{aligned}
$$

Therefore the variational freedom $|\delta \Psi\rangle$ should be constrained within the orthogonal complement of $|\Psi\rangle$ and $\left\{\left|\hat{G}_{i} \Psi\right\rangle\right\}_{i=1, M}$. Using projection operators, we can express such a variational freedom in the form

$$
\left.|\widetilde{\delta \Psi}\rangle=\left[\hat{1}-|\Psi\rangle\left\langle\Psi\left|-\sum_{i=1}^{M} \sum_{j=1}^{M}\right| \hat{G}_{i} \Psi\right\rangle\left(\boldsymbol{S}^{-1}\right)_{i j}\left\langle\hat{G}_{j} \Psi\right|\right]|\delta \Psi\rangle=\left[\hat{1}-|\Psi\rangle\langle\Psi|-\hat{P}^{G \Psi}\right] \mid \delta \Psi B 3\right)
$$


The variation of the expectation value of the Hamiltonian $\hat{H}$ with $\Psi$ is decomposed as

$$
\begin{aligned}
\delta\langle\Psi|\hat{H}| \Psi\rangle= & \langle\delta \Psi|\hat{H}| \Psi\rangle+\langle\Psi|\hat{H}| \delta \Psi\rangle \\
= & \langle\widetilde{\delta \Psi}|\hat{H}| \Psi\rangle+\langle\Psi|\hat{H}| \widetilde{\delta \Psi}\rangle \\
& \quad+\left[\left\langle\left[|\Psi\rangle\langle\Psi|+\hat{P}^{G \Psi}\right] \delta \Psi|\hat{H}| \Psi\right\rangle+\left\langle\Psi|\hat{H}|\left[|\Psi\rangle\langle\Psi|+\hat{P}^{G \Psi}\right] \delta \Psi\right\rangle\right](\mathrm{B} 4)
\end{aligned}
$$

It is sufficient that the VPSS extremal condition holds within the projected space $|\widetilde{\delta \Psi}\rangle$. Hence only the first terms in Eq. (B4) should disappear. The VPSS extremal condition is written as

$$
\left\langle\delta \Psi\left|\left[\hat{1}-|\Psi\rangle\langle\Psi|-\hat{P}^{G \Psi}\right] \hat{H}\right| \Psi\right\rangle=0, \quad \text { for }{ }^{\forall}\langle\delta \Psi|
$$

and Euler's equation in the projected space is

$$
\left[\hat{1}-|\Psi\rangle\langle\Psi|-\hat{P}^{G \Psi}\right] \hat{H}|\Psi\rangle=0
$$

Equation (B6) is just the same as Eq. (11) in the Lagrange multiplier method. 
[1] A. Messiah, Quantum Mechanics (Dover, New York, 2014).

[2] A. Mukherji and M. Karplus, J. Chem. Phys. 38, 44 (1963).

[3] H. Ichikawa and H. Kagawa, Int. J. Quantum Chem. 52, 575 (1994).

[4] Q. Wu and T. V. Voorhis, Phys. Rev. A 72, 24502 (2005).

[5] T. Ogawa, M. Sumita, Y. Shimodo, and K. Morihashi, Chem. Phys. Lett. 511, 219 (2011).

[6] K. Ohta, Chem. Phys. Lett. 329, 248 (2000).

[7] K. Ohta, Phys. Rev. A70, 022503 (2004).

[8] K. Ohta, Phys. Rev. A73, 044502 (2006).

[9] K. Ohta, Int. J. Quantum Chem. 113, 161 (2012).

[10] P. A. M. Dirac, Proc. Roy. Soc. A112, 673 (1926).

[11] P. A. M. Dirac, Proc. Cambridge Phil. Soc. 26, 376 (1930).

[12] P. M. Morse, H. Feshbach, Methods of Theoretical Physics (McGraw-Hill, New York, 1953).

[13] A. K. Kerman and S. E. Koonin, Ann. Phys. 100, 332 (1976).

[14] D. J. Rowe, A. R. Ryman, and G. Rosensteel, Phys. Rev. A22, 2362 (1980).

[15] P. Kramer and M. Saraceno, Geometry of the Time-Dependent Variational Principle in Quantum Mechanics (Springer, New York, 1981).

[16] K. -K. Kan, Phys. Rev. A24, 2831 (1981).

[17] J. B. Broeckhove, L. Lathouwers, E. Kesteloot, and P. Van Leuven, Chem. Phys. Lett. 149, 547 (1988).

[18] P. A. M. Dirac, Canad. J. Math., 2, 129 (1950).

[19] P. A. M. Dirac, Lectures on Quantum Mechanics (Yeshiva University Press, New York, 1964).

[20] E. C. G. Sudarshan and N. Mukunda, Classical Dynamics: A Modern Perspective (John Wiley \& Sons, New York, 1974).

[21] M. H. Henneaux and C. Teitelboim, Quantization of Gauge Systems (Princeton University Press, Princeton, New Jersey, 1992).

[22] J. O. Hirshfelfer, J. Chem. Phys. 33, 1462 (1960).

[23] S. T. Epstein and J. O. Hirschfelder, Phys. Rev. 123, 1495 (1961).

[24] A. Szabo and N. S. Ostlund, Modern Quantum Chemistry (Macmillan, New York, 1982).

[25] M. W. Schmidt, K. K. Baldridge, J. A. Boatz, S. T. Elbert, M. S. Gordon, J. H. Jensen, 
S. Koseki, N. Matsunaga, K. A. Nguyen, S. J. Su, T. L. Windus, M. Dupuis, and J. A. Montgomery, GAMESS, J. Comput. Chem. 14, 1347 (1993).

[26] C. C. J. Roothaan, Rev. Mod. Phys. 32, 179 (1960). 


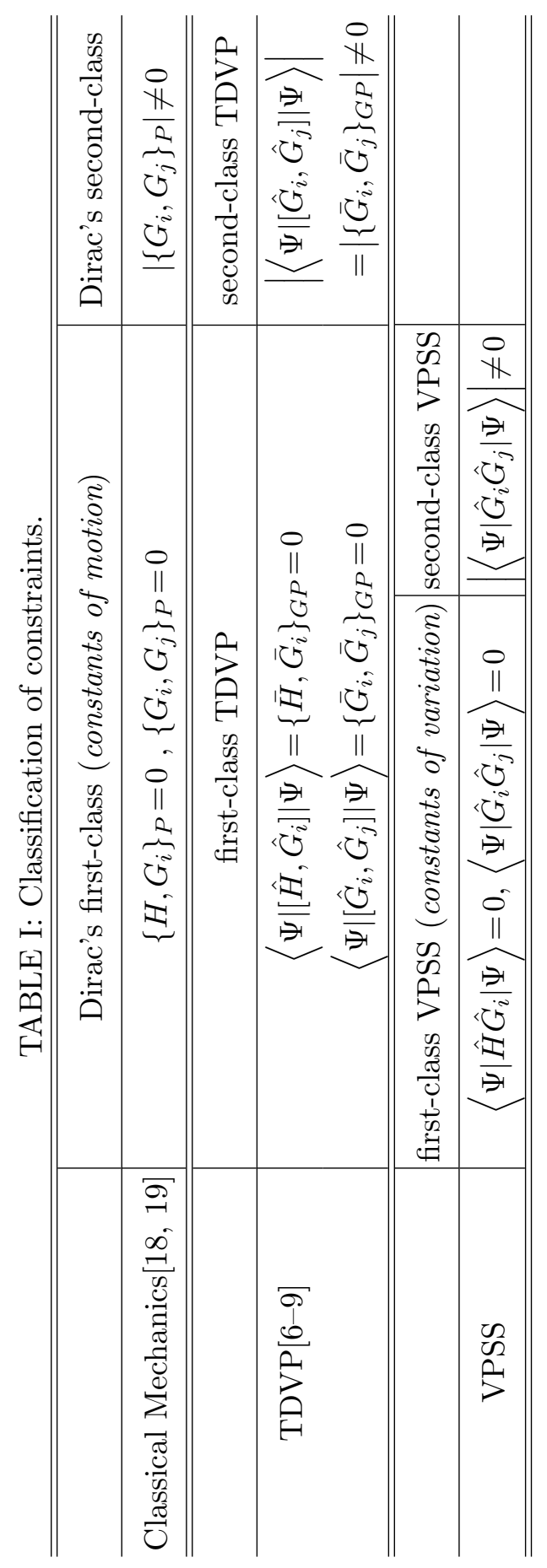




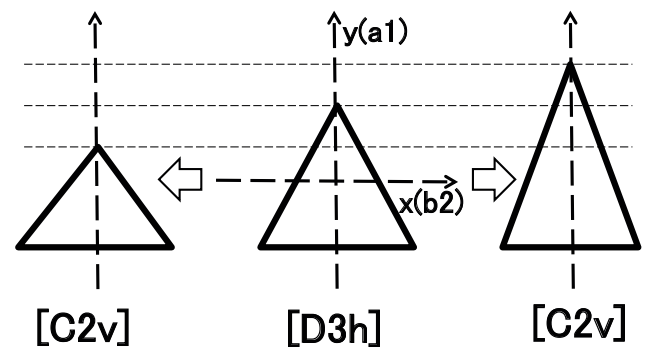

FIG. 1: $\mathrm{H}_{3}$ Jahn-Teller distortion from $D_{3 h}$ to $C_{2 v}$ by changing the height of the triangle.

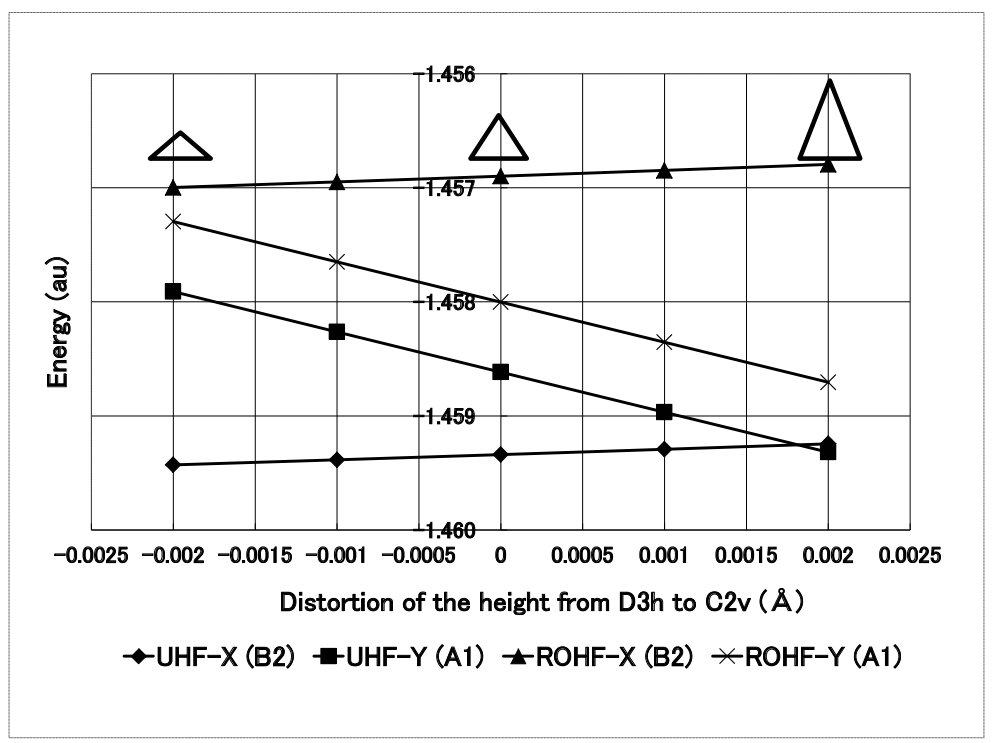

FIG. 2: $\mathrm{H}_{3}$ Jahn-Teller distortion by UHF and ROHF with DZV.

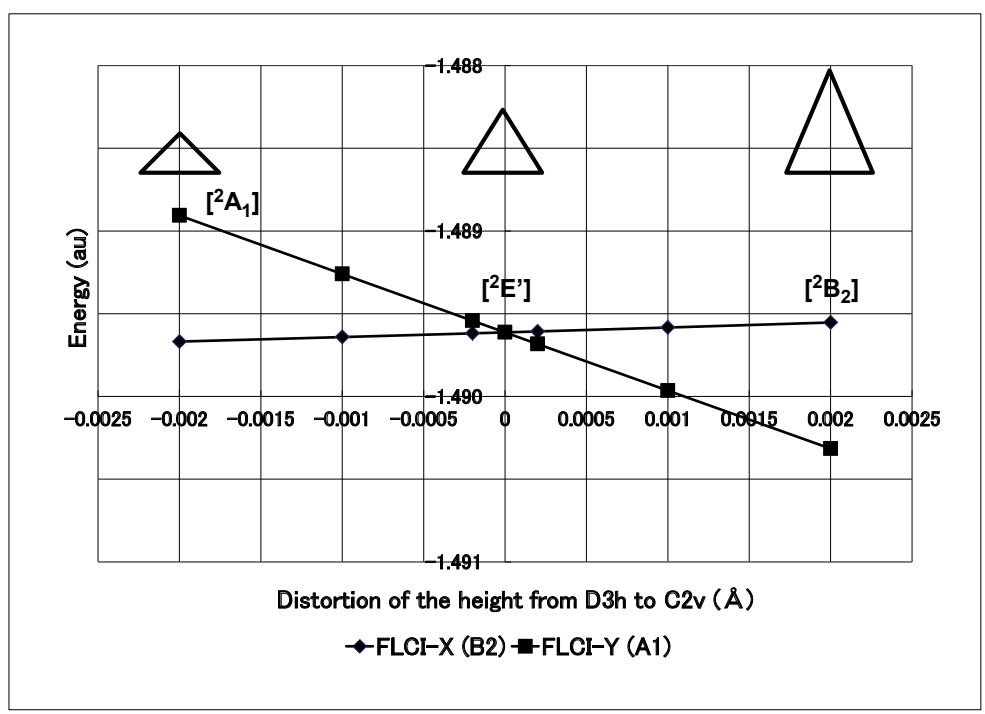

FIG. 3: $\mathrm{H}_{3}$ Jahn-Teller distortion by Full-CI with DZV. 